\title{
A Novel Efficient Simplified Strategy for MLFMA
}

\author{
Liu Zhanhe, Wang Rui, \\ School of Aeronautic Science and Engineering \\ Beijing University of Aeronautics and Astronautics \\ Beijing, China \\ nwpulzh@163.com
}

\author{
Bao Junbo, Wang Ying \\ School of Aeronautic Science and Engineering \\ Beijing University of Aeronautics and Astronautics \\ Beijing, China \\ Baojunbo117@163.com
}

\begin{abstract}
To solve large target RCS accurately and efficiently, based on CFIE (Combined Field Integral Equation), various factors influencing the memory, efficiency are analyzed in detail. Based on this analysis, a high-efficient and simple method used on solving large target RCS is proposed. By improving the comprehensive control and treatment on the gird, memory and precision of the algorithm, the aim that analyzing the electromagnetic scattering characteristic rapidly is realized under the condition of the effective resource. Numerical examples show that the simplified and efficient method is correct, and that other benefit such as improved computational efficiency and saved computational memory are obtained meanwhile the computational accuracy can be guaranteed.
\end{abstract}

Keywords-Electromagnetic scattering, Multilevel fast multipole algorithm, Radar cross section, aircraft

\section{INTRODUCTION}

Accurate calculation methods of electromagnetic scattering (such as MLFMA (multilevel fast multipole algorithm [1,2]), FDTD (finite difference time domain method) ) have become mainstream methods used in the RCS calculation of aircraft, weapons, tanks and other targets, as a substitute for the traditional high-frequency methods(such as physical optics, geometrical optics method, etc.). However, the accurate method is a generally lowfrequency method, which usually desires high memory and good hardware facilities. It can not even be used widely in some high frequency bands due to memory and other reasons, thus limiting the scope of its application. Take the current most rapid develop method, MLFMA for example, it can obtain high accuracy in the low frequency region and the resonance region. However, it can only calculate tenwavelength scattering targets and fail to calculate bigger ones because of high requirement of memory.

In response to above problems, there are two solutions. One is the parallel calculation method based on the network system or parallel computer computing platform. The network system is suitable for general analysis because of its low cost and convenient computation, while the parallel computer computing platform is not applicable to ordinary customers. The other method simplifies some approximation of certain physical quantities to save the memory from the viewpoint of the algorithm, which can bring about less accurate results. This method should not be used when the high calculation accuracy is required.
Based on the developed strategies, this paper analyzed various factors influencing the memory and efficiency of MLFMA: the form and parameters of the grid, cube grouping method, multipole mode number, stratification number and other factors. To improve computational efficiency further and reduce the memory effectively, a simplified and efficient calculation strategy was proposed. The RCS calculation of 100 -wavelength object in single computer was realized, which demonstrated this method was valid.

\section{THE BASIC THEORY OF MLFMA}

MLFMA has found extensive applications and has accelerated the moment of method (MOM) from $\mathrm{O}(\mathrm{N} 2)$ to $\mathrm{O}(\mathrm{N} \log \mathrm{N})$ for memory requirements and operation count, such as matrix multiplications[1-4], where $\mathrm{N}$ denotes the number of unknowns. When large-scale objects are considered and high computational efficiency is expected, the number $\mathrm{N}$ can be extremely large.

In this paper, electromagnetic scattering from perfect conductive objects, such as aircraft and missiles, is considered. The problem can be formulated in terms of combined field integral equation (CFIE) [1-5], and then the CFIE will be transformed into a linear matrix vector equation by modeling the object surface with patches and expanding the current in terms of rooftop basis functions. The basic principle behind Fast Multipole Method (FMM) is that CFIE is described as[4]:

$$
\begin{aligned}
& \mathbf{F}_{j}=\sum_{i=1}^{N} Z_{j i} a_{i}=\sum_{n \in \mathrm{G}_{\text {axx }}} \sum_{i \in \mathrm{G}_{n}} Z_{j i} a_{i}+\frac{i k}{4 \pi} \int d^{2} \hat{k} \mathbf{V}_{\text {finj }}(\hat{k}) . \\
& \sum_{n \in \mathrm{G}_{\mathrm{iti}}} \alpha_{m m}\left(\hat{r}_{m} \cdot \hat{k}\right) \sum_{i \in \mathrm{G}_{n}} \mathbf{V}_{\mathrm{sin}}^{*}(\hat{k}) a_{i}, \quad j \in \mathrm{G}_{m}
\end{aligned}
$$

Then the computation of matrix vector products is decomposed into two parts: the near interaction between adjacent sources and the far interaction between well separated ones, where $\alpha_{m n}$ and $a_{i}$ are the translation operator and the current factor, respectively, and $\mathrm{G}_{\text {near }}$ represents near groups while $\mathrm{G}_{\text {far }}$ represents far groups. $\mathbf{V}_{s i i}(\hat{k})$ and $\mathbf{V}_{f i n j}(\hat{k})$ are the radiation pattern and the receiving pattern, the other factors in (1) are found in [5].

FFM(Fast Multipole Method, FMM) on three-dimension scattering transforms surface integral equation into matrix equation, geometry mesh are gained in the scattering surface 
used the plane triangular surface element, curvilinear triangular surface method or curvilinear quadrilateral surface element method. The sub-group scattering are obtained through grouping and these groups are divided into near far groups according to the distance between the centers of each group.

In expressions (1), the near interaction is computed with MOM, while the far interaction is divided with MLFMA into three phases, namely the aggregation phase, the translation phase, and the disaggregation phase.

In the aggregation phase, the outgoing pattern of the finest level, which is described as $S_{L}$ (where $\mathrm{L}$ is the number of levels), is obtained by calculating the radiation patterns of corresponding sources. Then, the outgoing pattern of the coarser level is obtained by using interpolation and shifting from its child level. On the other hand, the incoming pattern of the top level is described as $B_{t}$, and that of the other level is obtained by anterpolation[1].

\section{MEMORY OCCUPATION ANALYSIS OF MLFMA}

MLFMA memory mainly includes three parts: block sparse matrix memory (S) for near group; the memory (V) of $\mathbf{V}_{s n i}(\hat{k})$ and $\mathbf{V}_{f n j}(\hat{k})$ for far group; the memory for the other parameters, such as the scattering surface geometry, transfer factor, interpolation matrix. The memory depends primarily on $\mathrm{S}, \mathrm{V}$ and geometric data (for large electrical size scattering body), of which the geometric data memory is only associated with the total number of unknowns $\mathrm{N}$.

For the sparse matrix memory, $S \propto c_{1} N_{b} N_{s}{ }^{2}=c_{1}(N)^{2} / N_{b}$, assuming uniform mesh, where NS denotes the number of unknowns in each group, $\mathrm{N}$ the total number of unknowns, $\mathrm{Nb}$ the total number of the cube in the finest layer, $\mathrm{cl}$ a constant number. It is shown that the block matrix correlates with the unknowns number and the relation is $\mathrm{O}(\mathrm{NbNS} 2)$.

The memory $\mathrm{V}$ can be divided into two parts, one is aggregation factor occupation memory $\mathrm{Vf}$ in the finest layer, and $V_{f} \propto c_{2} M_{f} N$, it is shown that the space complexity is $\mathrm{O}(\mathrm{NMf})$, where Mf denotes sampling points number of the thinnest layer, c2 a constant. The other is the total memory Val of aggregation and disaggregation factor through interpolation and extrapolation in all the layers. Assuming $\mathrm{Nlc}$ is the total number of all cube groups in the 1-th layer, so the memory $V_{l} \propto c_{3} N_{l c} M_{l}$ in this layer, and the order is $\mathrm{O}(\mathrm{NlcMl})$, where $\mathrm{Ml}$ is the number of sampling points. For the memory in all layers, $V_{a l}=\sum_{l} \sum_{k} V_{l k}=\sum_{l} V_{l}$.

\section{EFFICIENT SIMPLIFIED STRATEGY OF MLFMA}

Take the 1:1 aircraft model for example; the electrical size has reached 100-wavelength when the incident frequency is $3 \mathrm{GHz}$. For such a large size object, parallel methods and high-frequency algorithms are used to calculate a metallic sphere and conventional fighter model internationally at present. The calculation target in this paper is a high-stealth aircraft which has some features not available in conventional vehicles, such as low RCS (Radar Cross Section), smooth surface. For the low-RCS targets, it is necessary to employ the high-precision RCS calculation method.

For low RCS calculation of high-stealth configuration, the target with electrical size of 50-wavelength could be calculated through improvement and could obtain a high enough accuracy. When the electrical size further increased, the memory augmented dramatically and computational efficiency declined sharply, leading to the RCS of large electrical size object is almost impossible to solve, such as the usage of MLMFA to calculate target wavelength of 100 . Therefore, to ensure good enough precision, the great reduction of memory and significant improvement of efficiency become an important goal for electrically large scattering calculation.

For these requirements, according to all key parts of MLFMA, further simplification and improvement can greatly reduce the memory and ensure sufficient accuracy. Firstly, for the calculation of whole aircraft in $3 \mathrm{GHz}$ which belongs to high frequency scattering calculation. The electric size of the aircraft is 100 -wavelength, which far exceeds the current handling capacity of MLFMA. Secondly, for the scattering calculation of high frequency stealth layout, its low RCS demands higher accuracy requirement of computational method. From the above two points, according to the law of memory occupation and control of MLFMA and the requirement of certain accuracy, a novel efficient simplified strategy is proposed as follows:

The first step simplification: the ratio of wavelength and grid size was reduced appropriately to $1 / 6$ or even higher, which needs to ensure the accuracy of surface, by using the second rectangular grid, thus the memory of sparse matrix for the near groups was reduced; meanwhile, in aggregation/disaggregation phase, the cube size in the thinnest layer can be selected to be $0.5 \sim 1.2$ times wavelength according to the practical conditions.

The second step simplification: multipole model number $(\mathrm{MMN})$, integral sampling points number and layer number were declined appropriately for aggregation/disaggregation. However, it was necessary to ensure the MMN and interpolation nodes in the finest layer to avoid the decrease of accuracy. Through the second step simplification, memory occupation of aggregation/disaggregation can be adjusted to obtain less resource consumption and high calculation efficiency。

Through the above simplification strategy, it can be seen that the main portion of memory could be reduced greatly by self-control. On the other hand, the parameter settings influencing the accuracy were considered in order to ensure computational accuracy.

Meanwhile, we synthesized high-precision control technology, the memory control technology and model number control technology, combined the division criteria and method of grid size and controlled the ratio between grid size and the incident wavelength, thus controlling different calculation states dynamically and comprehensively. 


\section{NUMERICAL EXAMPLES AND ANALYSIS}

In this paper, we employed MLFMA to complete the $3 \mathrm{GHz}$ RCS calculation of high-stealth whole aircraft model under the conditions of the electric size of 100-wavelength. The computing grid of the aircraft model is as follows:

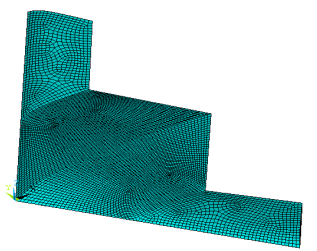

Figure 1. The sketch of an aircraft grid

Based on the second rectangular grid, we applied the proposed high efficient simplified method and set the ration between the incident wave length and the grid geometry as $1 / 5$ and the side length of thinnest layer cube 0.8 times wavelength. Meanwhile, we calculated the MMN accurately in each layer[5 7], reduced the MMN appropriately and decreased the integral sampling points, which effectively reduced the memory occupation.

For the aircraft model shown in Figure 2, when the incident frequency is $3 \mathrm{GHz}$ and the unknown number is 252,247 calculated in a $3.0 \mathrm{G} \mathrm{CPU}$ dual-core PC, the memory reduced from original $6.2 \mathrm{~GB}$ to $1.46 \mathrm{~GB}$. the RCS curves is as follows:

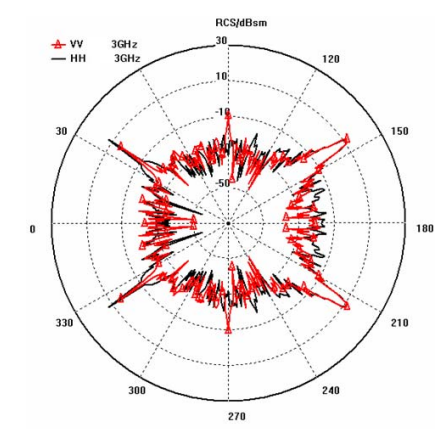

Figure 2. The RCS curves of an aircraft

It can be seen from the RCS curve, using the new rapid solution strategy, the calculated curve trend is right for horizontal and vertical polarization, and the curve peaks and valleys of head, lateral and tail conform to the shape design of stealth aircraft, which show that the calculation method is effective.

Meanwhile, this numerical example also illustrated that it could successfully achieved the electromagnetic scattering calculations of electrical size above 100-wavelength objects in single computer by this proposed method (before improvement, the single computer only handled electrical size about 30-wavelength objects), and that it had more advantages compared with parallel computing .

\section{CONCLUSION}

For the MLFMA, to improve the computational efficiency, from the characteristic of memory allocation and control, a new high-efficient simplified strategy aim at improving the computational efficiency and reducing the memory was proposed. Based on this method, electromagnetic scattering analysis of large-scale electrical objects could be computed, thus improving the calculation efficiency and saving computing resources, which provided technical possibilities to analyze the electromagnetic scattering characteristics.

\section{REFERENCES}

[1] J M Song, C C Lu, W C Chew. Multilevel fast multipole algorithm for electromagnetic scattering by large complex objects. IEEE Transaction on Antennas Propagation[J], 1997, 45(3):1488-1493.

[2] J M Song, W C Chew. Fast multipole method solution of three dimensional integral equation. IEEE Trans. on Antennas Propagation[J], 1995, 45(10): 1528-1531.

[3] J M Song, W C Chew, Moment method solution using parametric geometry, Journal of Electromagnetic Waves and Applications[J], 1995, 9(1):71-83.

[4] Z H Liu, P L Huang, Z Wu, X Gao. Improvement and performance of parallel multilevel fast multipole algorithm," Journal of Systems Engineering and Elctronics[J], 2011,22(1):164-169.

[5] Z H Liu, P L Huang, X Gao, Y Li, J Z Ji, Multi-frequency RCS reduction characteristics of shape stealth with MLFMA with improved MMN. Chinese journal of aeronautics[J], 2010, 23(3):327333.

[6] Z H Liu, Z Wu, J Zhou, X Gao. Improving multilevel fast mutlipole algorithm. ACTA Aeronautical ET Astronautical SINICA, 2008, 29(5):1180-1185.

[7] J Wen, J Hu, Z P Nie. A novel st rategy of the multipole numbers of $t$ he ML FMA [ C ] \| Digital Object Identifier APMC. Piscataway : IEEE, $2005: 427$. 